Nuclear Physics B (Proc. Suppl.) 25A (2018) 1-10

North-Holland

\title{
A PROPOSAL FOR SIMULATING CHIRAL FERMIONS *
}

\author{
M. Göckeler ${ }^{1,2}$ and G. Schierholz ${ }^{2,3}$ \\ 1 Institut für Theoretische Physik, RWTH Aachen, \\ Sommerfeldstraße, D-5100 Aachen, Germany \\ ${ }^{2}$ Gruppe Theorie der Elementarteilchen, Höchstleistungsrechenzentrum HLRZ, \\ c/o KFA, Postfach 1913, D-5170 Jülich, Germany \\ 3 Deutsches Elektronen-Synchrotron DESY, \\ Notkestraße 85, D-2000 Hamburg 52, Germany
}

\begin{abstract}
We describe a method for evaluating chiral gauge theories that is not plagued by the doubling problem. To
\end{abstract} demonstrate the efficiency of the approach, we apply our ideas to the chiral Schwinger model.

\section{INTRODUCTION}

Chiral gauge theories play an important role in particle physics. Yet very little is known about their properties. The main reason for this is the lack of a calculational method which takes into account non-perturbative effects.

The lattice regularization was invented for that purpose. Until now we have, however, not been able to find a consistent lattice formulation of these theories. Due to the doubling problem one is always led to an equal number of left- and right-handed fermions with net chirality zero. Attempts to remove the unwanted "doublers" by pushing their masses to the cutoff while preserving chiral symmetry have, in

* TALK GIVEN AT THE WORKSHOP ON NONPERTURBATIVE ASPECTS OF CHIRAL GAUGE THEORIES, ROME, MARCH 1992 spite of great efforts, not brought the desired success [1]. Some authors have arranged themselves with this situation by postulating the existence of mirror fermions [2]. But even these models are inadequate. A non-vector theory leads after the anomalies have been cancelled - to a gauge invariant, complex effective action whose imaginary part is non-zero [3]. The action of the mirror-fermion model is, however, real.

But it may also be that non-vector theories do not exist as consistent field theories with nontrivial dynamics. A first answer to this question can be obtained from the chiral Schwinger model. In four dimensions one has to resort to numerical methods to address this problem. We believe, however, that it should at least be possible to answer some of the more fundamental questions, in particular whether the imaginary part of the effective action is indeed non-zero. 
In order to evade the doubling problem, we propose to evaluate the fermionic action in the background of continuum gauge fields, which we will construct from lattice gauge field configurations. The starting point is a formula for the effective action, which was derived some time ago by Alvarez-Gaumé et al. [3] for a similar purpose. The virtue of this formula is that it involves the ordinary covariant Dirac operator only and that it is amenable to explicit calculations. The basic idea of the method as well as some theoretical background are presented in sec. 2. In sec. 3 we sketch the derivation of continuum gauge fields from lattice gauge field configurations. Our ideas are then applied to the chiral Schwinger model in sec. 4 . Finally, in sec. 5 we present some concluding remarks.

\section{EFFECTIVE ACTION}

We will work in Euclidean space compactified to the torus $\mathbf{T}^{4}$. But one can also think of other geometries. Consider now the Dirac operator

$i \not D_{\mp}(A)=i\left(\not \partial+\not A P_{\mp}\right), P_{\mp}=\frac{1 \mp \gamma_{5}}{2}$,

which minimally couples left-handed and righthanded fermions, respectively, to the gauge fields. The gauge fields are assumed to be in a complex representation of some gauge group $G$,

$A_{\mu}=A_{\mu}^{a} T^{a}, A_{\mu}^{\dagger}=-A_{\mu}$.

We are interested in the effective action for lefthanded fermions, $S_{-}(A)$. This is given by

$e^{-S_{-}(A)}=\int \mathcal{D} \bar{\psi} \mathcal{D} \psi e^{-\bar{\psi} i \not D_{-}(A) \psi}$.

It is understood that eq. (3) and the following expressions are properly regularized, without com- mitting ourselves to any particular scheme for the moment. For the real part of $S_{-}(A)$ one finds

$2 \operatorname{Re} S_{-}(A)=S_{-}(A)+S_{+}(A)=S(A)+S(0)$

with

$e^{-S(A)}=\int \mathcal{D} \bar{\psi} \mathcal{D} \psi e^{-\bar{\psi} i \not D(A) \psi}$,

$i \not D(A)=i(\not \partial+\not A)$,

which means that it is given by the action of the corresponding vector theory. This part of the action does not constitute any problems. It can be computed by standard lattice techniques.

Thus the chiral nature of the fermions is reflected entirely in the imaginary part of the effective action. Following Alvarez-Gaumé et al. [3] we shall now derive an expression for $\operatorname{Im} S_{-}(A)$ on which we will base our further discussion. Our derivations will be purely formal. For rigorous proofs the reader is refered to the original literature [3]. We start from the expression

$$
\begin{aligned}
& S_{-}(A)-S_{-}(0) \\
& =-\ln \operatorname{det} i \not D_{-}(A)+\ln \operatorname{det} i \not D_{-}(0) \\
& =-\operatorname{Tr} \ln i \not D_{-}(A)+\operatorname{Tr} \ln i \not D_{-}(0) \\
& =-\operatorname{Tr} \ln \left(1+(i \not \partial)^{-1} i \not A P_{-}\right) .
\end{aligned}
$$

Expanding the logarithm, this gives

$$
\begin{aligned}
& S_{-}(A)-S_{-}(0) \\
& =\operatorname{Tr} \sum_{n=1}^{\infty} \frac{(-1)^{n}}{n}\left((i \not \partial)^{-1} i \not A\right)^{n} P_{-} .
\end{aligned}
$$

By replacing $A$ by ${ }^{t} A=t A$ on the right-hand side of eq. (8), differentiating the whole expression with respect to $t$ and integrating over $t$ again, one obtains after doing the sum over $n$ again 


$$
\begin{aligned}
& S_{-}(A)-S_{-}(0) \\
& =-\int_{0}^{1} d t \operatorname{Tr} i \not A\left(i \not D\left({ }^{t} A\right)\right)^{-1} P_{+} .
\end{aligned}
$$

This results in the expression for the imaginary part

$\operatorname{Im} S_{-}(A)=-\frac{1}{2} \int_{0}^{1} d t \operatorname{Tr} \gamma_{5} \not A\left(i \not D\left({ }^{t} A\right)\right)^{-1}$.

While the real part of the action is gauge invariant, the imaginary part is generally not. Under gauge transformations

$A_{\mu} \rightarrow^{g} A_{\mu}=g^{-1}\left(A_{\mu}+\partial_{\mu}\right) g$

it transforms like

$\operatorname{Im} S_{-}\left({ }^{g} A\right)-\operatorname{Im} S_{-}(A)=2 \pi Q_{5}$,

where $Q_{5}$ is the Wess-Zumino action. If the theory is anomaly-free, $Q_{5}=0$ and $S_{-}(A)$ is gauge invariant. But we still expect $\operatorname{Im} S_{-}(A) \neq 0$, also in a model with mirror fermions.

A straightforward lattice calculation of eq. (10) with, e.g., Wilson fermions would give a divergent result in the continuum limit. The situation is similar to the case of the topological charge,

$Q=-\frac{1}{16 \pi^{2}} \int d^{4} x \operatorname{Tr} F \tilde{F}$,

$F \tilde{F}=\frac{1}{2} \epsilon_{\mu \nu \rho \sigma} F_{\mu \nu} F_{\rho \sigma}$,

where the naive latice transcription of $F \tilde{F}$, A does not have any topological significance. Indeed, it has been shown [3] that the gauge invariant part of $\operatorname{Im} S_{-}(A)$ is given by the $\eta$-invariant of the five-dimensional Dirac operator,

$H=i \gamma_{5} \partial_{t}+i \not D\left({ }^{t} A\right)$, which is a topological invariant.

Let us stay with the example of the topological charge for a moment. It is well known that the naive expression for $Q$ receives perturbative contributions, which are divergent in the continuum limit and so cover all topological effects. The origin of these contributions lies in fluctuations of the gauge fields on the scale of the lattice spacing. These fluctuations are unphysical near the continuum limit, and only those theories which have a continuum limit are considered here. If we now extrapolate the lattice gauge field smoothly to the interior of the hypercubes - thereby respecting the fact that a topologically non-trivial gauge field configuration cannot be in the same gauge everywhere on the torus - and calculate $Q$ in the background of this continuum gauge field using the expression (13), we find not only that all divergences have been eliminated $[\mathbb{}$, but also that $Q$ is an integer as it should. (See the next section for further details.) This is the essential idea behind the geometrical expressions for the topological charge [6, 7,8], though the actual calculation of $Q$ proceeds via the transition functions, which connect the gauges in the different hypercubes, and for those it is sufficient to know the gauge field on the boundary of the hypercubes only. Given the continuum gauge field, it would equally well be possible to compute the topological charge by discretizing this field again, but on a finer mesh, computing the corresponding link matrices and using the naive lattice expression for $F \tilde{F}$ [ ] th obtain $Q$. The continuum value of the charge is then found by making the mesh finer and finer until the result has converged.

We now propose to compute $\operatorname{Im} S_{-}(A)$ in an analogous way. We start from a lattice gauge field configuration, which we regard for the mo-

\#1 We assume that the action has been chosen such that the susceptibility is not affected by dislocations [5]. 
ment as being given to us. The first problem is then to extrapolate the lattice gauge field to the whole interior of the hypercube. This construction, which is highly non-trivial, will be sketched in the next section. Further details can be found in the original publication [9]. The next problem is to compute $\operatorname{Im} S_{-}(A)$ in the background of this field. As we have already indicated, the expressions for the effective action have to be properly regularized in order to make sense. We suggest to use the lattice regularization with a lattice spacing, $a$, much smaller than the original lattice spacing. The latter we shall set equal to one for convenience. For Wilson fermions we then find

$$
\begin{aligned}
& \operatorname{Im} S_{-}(A)=\frac{i}{2} \int_{0}^{1} d t a^{4} \sum_{x, \mu}\left\{\operatorname{Tr} \gamma_{5}\left(\gamma_{\mu}-1\right)\right. \\
& \times \frac{1}{2 a} \ln \mathcal{U}_{x, \mu} \mathcal{U}_{x, \mu}^{t} \mathcal{G}_{x+\hat{\mu}, x}+\operatorname{Tr} \gamma_{5}\left(\gamma_{\mu}+1\right) \\
& \left.\times \frac{1}{2 a} \ln \mathcal{U}_{x, \mu} \mathcal{U}_{x, \mu}^{-t} \mathcal{G}_{x, x+\hat{\mu}}\right\}
\end{aligned}
$$

where $\mathcal{U}$ are the link matrices on the fine sublattice, and $\mathcal{G}$ is the inverse fermion matrix,

$$
\begin{aligned}
& \frac{1}{2 a} \sum_{\mu}\left(\gamma_{\mu}-1\right) \mathcal{U}_{x, \mu}^{t} \mathcal{G}_{x+\hat{\mu}, y} \\
& -\frac{1}{2 a} \sum_{\mu}\left(\gamma_{\mu}+1\right) \mathcal{U}_{x-\hat{\mu}, \mu}^{-t} \mathcal{G}_{x-\hat{\mu}, y} \\
& \left.+\frac{4}{a} \mathcal{G}_{x, y}\right\}=\frac{1}{a^{4}} \delta_{x, y} \mathbf{1} .
\end{aligned}
$$

It is straightforward to compute the link matrices $\mathcal{U}$, as well as the logarithm and powers of $\mathcal{U}$, from the continuum gauge field. The right-hand side of eq. (16) can be very efficiently calculated by means of a stochastic estimator 10]. This requires only one inversion of the fermion matrix for one set of random numbers. In the final step we then have to perform the calculations on finer and finer sublattices until we obtain a stable result (if this exists).
We like to emphasize that eq. (16) is only one out of many possibilities of computing $\operatorname{Im} S_{-}(A)$ in the background of a continuum field. An alternative method would be to use the gauge field $A_{\mu}(x)$ directly instead of $\ln \mathcal{U}_{x, \mu} \mathcal{U}_{x, \mu}^{t}$. But this would require a further regularization step. One such step is the point-splitting technique. Which method is best suited can only be decided in concrete calculations which we will return to in the future.

It may be possible that there are more elegant ways of computing the imaginary part of the effective action. Alvarez-Gaumé has attempted a geometric construction similar to that of the topological charge, but without success [11. In two dimensions it turns out that one can calculate the effective action analytically, as we will show in sec. 4 .

Whether we can do simulations of chiral gauge theories depends now on the magnitude of $\operatorname{Im} S_{-}(A)$. So far we do not know how to handle complex actions. If $\operatorname{Im} S_{-}(A)$ is small, we may simulate gauge field configurations according to the real part of the effective action (including the gauge field action) on the lattice in the standard fashion and include the phase factor in the statistical average over the ensemble.

\section{CONTINUUM GAUGE FIELDS}

We shall now sketch the construction of continuum gauge fields $A_{\mu}(x)$ from lattice gauge field configurations. We work on a hypercubic lattice with periodic boundary conditions. The lattice points are denoted by $s$, and the link matrices are called $U_{s, \mu}$.

The gauge fields have to fulfill the following constraints. (i) The link matrices derived from 
$A_{\mu}$ must agree with those of the original lattice, $U_{s, \mu}$. (ii) The construction must be gauge covariant, which means that a lattice gauge field transformation must result in a continuum gauge field transformation of $A_{\mu}(x)$. (iii) The gauge field $A_{\mu}(x)$ should lead to the fiber bundle which has been previously derived from the lattice gauge field configuration [6]. This guarantees that the topological charge is the same in both cases.

In the first step we construct in each hypercube,

$c(s)=\left\{x \in \mathbf{T}^{4} \mid s_{\mu} \leq x_{\mu} \leq s_{\mu}+1, \forall \mu\right\}$,

a local gauge field $A_{\mu}^{(s)}(x)$, such that on the intersection of two adjacent hypercubes the corresponding gauge fields are connected by a gauge transformation given by Lüscher's transition functions [6]. In the second step we then construct a global, and generally singular, gauge field out of the local gauge fields.

Because the construction involves derivatives of the transition functions in intermediate steps, it is necessary to enlarge the hypercubes to open sets,

$\tilde{c}(s)=\left\{x \in \mathbf{T}^{4} \mid s_{\mu}-\epsilon<x_{\mu}<s_{\mu}+1+\epsilon, \forall \mu\right\}$,

and define smeared transition functions on open faces $\tilde{f}(s, \mu)=\tilde{c}(s) \cap \tilde{c}(s-\hat{\mu})$ :

$$
\begin{aligned}
& \tilde{v}_{s, \mu}(x)=v_{s, \mu}\left(s_{1}+\phi\left(x_{1}-s_{1}\right), \cdots\right. \\
& \left.\cdots, s_{\mu}, \cdots, s_{4}+\phi\left(x_{4}-s_{4}\right)\right) .
\end{aligned}
$$

Here $v_{s, \mu}$ is Lüscher's transition function [6] defined on $f(s, \mu)=c(s) \cap c(s-\hat{\mu})$ and $\phi$ is a smooth function, $\phi: \mathbf{R} \rightarrow[0,1]$, with the properties

$$
\phi(t)= \begin{cases}0 & \text { for } t<\eta \\ 1 & \text { for } t>1-\eta\end{cases}
$$

and $\epsilon<\eta<1 / 2$. It follows that the $\tilde{v}$ 's satisfy the cocycle condition

$\tilde{v}_{s-\hat{\mu}, \nu}(x) \tilde{v}_{s, \mu}(x)=\tilde{v}_{s-\hat{\nu}, \mu}(x) \tilde{v}_{s, \nu}(x)$

on $\tilde{p}(s, \mu, \nu)=\tilde{f}(s, \mu) \cap \tilde{f}(s-\hat{\nu}, \mu)$ and therefore define a principal bundle over $\mathbf{T}^{4}$. The gauge field $A_{\mu}^{(s)}(x)$ can now be constructed step by step making use of the relation

$$
\begin{aligned}
& A_{\mu}^{(s-\hat{\nu})}(x)=\tilde{v}_{s, \nu}(x) A_{\mu}^{(s)}(x) \tilde{v}_{s, \nu}^{-1}(x) \\
& +\tilde{v}_{s, \nu}(x) \partial_{\mu} \tilde{v}_{s, \nu}^{-1}(x) .
\end{aligned}
$$

Starting from the value zero at $x \in \tilde{c}(s),-\epsilon<$ $x_{\nu}-s_{\nu}<\epsilon$ for $\forall \nu$, eq. 23) gives the gauge field in the neighborhoods of the other corners of $\tilde{c}(s)$, where it is also zero. In the next step one takes the gauge field to be zero in $\epsilon$-neighborhoods of the links in $\tilde{c}(s)$ which originate from $s$ and applies eq. (23) again. Then one defines $A_{\mu}^{(s)}(x)$ on the six plaquettes $\tilde{p}(s, \mu, \nu)$ by interpolating the expressions already obtained. By means of eq. (23) one finds $A_{\mu}^{(s)}(x)$ on all the other plaquettes contained in $\tilde{c}(s)$. Continuing in this fashion one arrives at the expression

$$
\begin{aligned}
& A_{\mu}^{(s)}(x)=\phi\left(x_{\alpha}-s_{\alpha}\right) \phi\left(x_{\beta}-s_{\beta}\right) \phi\left(x_{\gamma}-s_{\gamma}\right) \\
& \times\left\{Z_{\mu}\left(s, \alpha, \beta, \gamma \mid s_{\alpha}+1, s_{\beta}+1, s_{\gamma}+1, x_{\mu}\right)\right. \\
& +\sum_{\substack{\text { cycl. perm. } \\
(\alpha, \beta, \gamma)}}\left[-\operatorname{Ad}\left(\left(\tilde{v}_{s+\hat{\alpha}, \alpha}\right.\right.\right. \\
& \left.\left.\times \tilde{v}_{s+\hat{\alpha}+\hat{\beta}, \beta}\right)\left(s_{\alpha}+1, s_{\beta}+1, x_{\gamma}, x_{\mu}\right)\right) \\
& \times Z_{\mu}\left(s+\hat{\alpha}+\hat{\beta}, \gamma \mid s_{\alpha}+1, s_{\beta}+1, s_{\gamma}+1, x_{\mu}\right) \\
& +\operatorname{Ad}\left(\tilde{v}_{s+\hat{\alpha}, \alpha}\left(s_{\alpha}+1, x_{\beta}, x_{\gamma}, x_{\mu}\right)\right. \\
& \left.\times \tilde{v}_{s+\hat{\alpha}+\hat{\beta}, \beta}\left(s_{\alpha}+1, s_{\beta}+1, x_{\gamma}, x_{\mu}\right)\right) \\
& \times Z_{\mu}\left(s+\hat{\alpha}+\hat{\beta}, \gamma \mid s_{\alpha}+1, s_{\beta}+1, s_{\gamma}+1, x_{\mu}\right)
\end{aligned}
$$




$$
\begin{aligned}
& +\operatorname{Ad}\left(\tilde{v}_{s+\hat{\alpha}, \alpha}\left(s_{\alpha}+1, x_{\beta}, x_{\gamma}, x_{\mu}\right)\right. \\
& \left.\times \tilde{v}_{s+\hat{\alpha}+\hat{\gamma}, \gamma}\left(s_{\alpha}+1, x_{\beta}, s_{\gamma}+1, x_{\mu}\right)\right) \\
& \times Z_{\mu}\left(s+\hat{\alpha}+\hat{\gamma}, \beta \mid s_{\alpha}+1, s_{\beta}+1, s_{\gamma}+1, x_{\mu}\right) \\
& -\operatorname{Ad}\left(\tilde{v}_{s+\hat{\alpha}, \alpha}\left(s_{\alpha}+1, x_{\beta}, x_{\gamma}, x_{\mu}\right)\right) \\
& \left.\left.\times Z_{\mu}\left(s+\hat{\alpha}, \beta, \gamma \mid s_{\alpha}+1, s_{\beta}+1, s_{\gamma}+1, x_{\mu}\right)\right]\right\} \\
& +\sum_{\substack{\text { cycl. perm. } \\
(\alpha, \beta, \gamma)}}\left[-\phi\left(x_{\alpha}-s_{\alpha}\right) \phi\left(x_{\beta}-s_{\beta}\right)\right. \\
& \times Z_{\mu}\left(s, \alpha, \beta \mid s_{\alpha}+1, s_{\beta}+1, x_{\gamma}, x_{\mu}\right) \\
& +\phi\left(x_{\alpha}-s_{\alpha}\right) \phi\left(x_{\beta}-s_{\beta}\right) \\
& \times \operatorname{Ad}\left(\tilde{v}_{s+\hat{\alpha}, \alpha}\left(s_{\alpha}+1, x_{\beta}, x_{\gamma}, x_{\mu}\right)\right) \\
& \times Z_{\mu}\left(s+\hat{\alpha}, \beta \mid s_{\alpha}+1, s_{\beta}+1, x_{\gamma}, x_{\mu}\right) \\
& +\phi\left(x_{\alpha}-s_{\alpha}\right) \phi\left(x_{\gamma}-s_{\gamma}\right) \\
& \times \operatorname{Ad}\left(\tilde{v}_{s+\hat{\alpha}, \alpha}\left(s_{\alpha}+1, x_{\beta}, x_{\gamma}, x_{\mu}\right)\right) \\
& \times Z_{\mu}\left(s+\hat{\alpha}, \gamma \mid s_{\alpha}+1, x_{\beta}, s_{\gamma}+1, x_{\mu}\right) \\
& \left.+\phi\left(x_{\alpha}-s_{\alpha}\right) Z_{\mu}\left(s, \alpha \mid s_{\alpha}+1, x_{\beta}, x_{\gamma}, x_{\mu}\right)\right] \text {, }
\end{aligned}
$$

where

$$
\begin{aligned}
& Z_{\mu}(s, \alpha, \beta, \gamma \mid x) \\
& =\left(\tilde{v}_{s+\hat{\gamma}, \gamma} \tilde{v}_{s+\hat{\gamma}+\hat{\beta}, \beta} \tilde{v}_{s+\hat{\gamma}+\hat{\beta}+\hat{\alpha}, \alpha}\right. \\
& \left.\times \partial_{\mu} \tilde{v}_{s+\hat{\gamma}+\hat{\beta}+\hat{\alpha}, \alpha}^{-1} \tilde{v}_{s+\hat{\gamma}+\hat{\beta}, \beta}^{-1} \tilde{v}_{s+\hat{\gamma}, \gamma}^{-1}\right)(x), \\
& Z_{\mu}(s, \alpha, \beta \mid x)=\left(\tilde{v}_{s+\hat{\alpha}, \alpha} \tilde{v}_{s+\hat{\alpha}+\hat{\beta}, \beta}\right. \\
& \left.\times \partial_{\mu} \tilde{v}_{s+\hat{\alpha}+\hat{\beta}, \beta}^{-1} \tilde{v}_{s+\hat{\alpha}, \alpha}^{-1}\right)(x), \\
& Z_{\mu}(s, \alpha \mid x)=\left(\tilde{v}_{s+\hat{\alpha}, \alpha} \partial_{\mu} \tilde{v}_{s+\hat{\alpha}, \alpha}^{-1}\right)(x), \\
& \operatorname{Ad}(\tilde{v}) M=\tilde{v} M \tilde{v}^{-1} .
\end{aligned}
$$

Note that $Z_{\mu}(s, \alpha, \beta, \gamma \mid x)$ and $Z_{\mu}(s, \alpha, \beta \mid x)$ are symmetric in $\alpha, \beta, \gamma$ and $\alpha, \beta$, respectively, due to the cocycle condition. It is straightforward to verify that for $x \in \tilde{f}(s, \mu)$

$$
\left.\mathcal{P} \exp \left\{\int_{0}^{1} d t A_{\mu}^{(s)}(x+(1-t) \hat{\mu})\right\}\right]=u_{x, x+\hat{\mu}}^{s}
$$

where $u_{x, x+\hat{\mu}}^{s}$ are the link variables in the complete axial gauge defined in ref. [6]. In eq. (24) one can take the limit $\epsilon \rightarrow 0$ and put $\phi(t)=t$ for $0 \leq t \leq 1$.

In order to obtain the global gauge field we make use of the section $w^{s}$, originally defined on the boundary of the hypercube [12] and extended to its interior in ref. [9], and write

$$
\begin{aligned}
& A_{\mu}(x)=w^{s}(x)^{-1} A_{\mu}^{(s)}(x) w^{s}(x) \\
& +w^{s}(x)^{-1} \partial_{\mu} w^{s}(x)
\end{aligned}
$$

Under a lattice gauge transformation,

$U(s, \mu) \rightarrow \bar{U}(s, \mu)=g(s) U(s, \mu) g(s+\hat{\mu})^{-1}$,

the local gauge field transforms as

$\bar{A}_{\mu}^{(s)}(x)=g(s)^{-1} A_{\mu}^{(s)}(x) g(s)$,

and the section transforms as [9]

$\bar{w}^{s}(x)=g(s) w^{s}(x) g(x)^{-1}$,

where $g(x)$ is the lattice gauge transformation extrapolated to the interior of all the hypercubes. The latter is given in ref. [9]. This gives the desired result

$\bar{A}_{\mu}(x)=g(x)\left(A_{\mu}(x)+\partial_{\mu}\right) g(x)^{-1}$.

\section{AN EXAMPLE: THE CHIRAL SCHWINGER MODEL}

We shall now apply our method to the chiral Schwinger model. A lot is known about this 
model from other studies [13]. Our approach differs from most of the other approaches by working in Euclidean space. Questions regarding the positivity of the transfer matrix and the like will and can be ignored here.

The action of this model is

$$
\int d^{2} x \bar{\psi} i\left(\not \partial+\not A P_{-}\right) \psi
$$

Our choice of the $\gamma$ matrices is

$\gamma_{1}=\left(\begin{array}{ll} & 1 \\ 1 & \end{array}\right), \gamma_{2}=\left(\begin{array}{cc} & -i \\ i & \end{array}\right)$,

with

$$
\gamma_{5}=i \gamma_{1} \gamma_{2}=\left(\begin{array}{ll}
-1 & \\
& 1
\end{array}\right) .
$$

The gauge fields will be written $A_{\mu}=i A_{\mu}^{0}$, where $A_{\mu}^{0}$ is real. This then leads to the Dirac operator

$$
\begin{aligned}
i \not D(A) & =i\left(\begin{array}{cc} 
& \partial_{1}+A_{2}^{0} \\
\partial_{1}-A_{2}^{0} &
\end{array}\right) \\
& +\left(\begin{array}{cc}
-\partial_{2}-A_{1}^{0} & \partial_{2}-A_{1}^{0}
\end{array}\right) .
\end{aligned}
$$

We may assume that $i \not D(A)$ has no zero modes due to the vanishing of $\operatorname{det} i \not D(A)$ in this case. Being a two-dimensional model, it is easy to find a closed form for the inverse Dirac operator in eqs. (9.10), which is the solution of

$i \not D(A)(i \not D(A))^{-1}(x, y)=\delta^{2}(x-y) \mathbf{1}$.

We obtain

$$
\begin{aligned}
& (i \not D(A))^{-1}(x, y)=-\int \frac{d^{2} k}{(2 \pi)^{2}} \frac{\mathrm{e}^{i k(x-y)}}{k^{2}} \\
& \times \not k\left(\begin{array}{cc}
f^{*-1}(x, y) & f(x, y)
\end{array}\right),
\end{aligned}
$$

where

$$
\begin{aligned}
& f(x, y)=\exp \left\{\int d^{2} z(s(x, z)-s(y, z))\right. \\
& \left.\times\left(A_{1}^{0}(z)+i A_{2}^{0}(z)\right)\right\}
\end{aligned}
$$

with

$s(x, z)=\int \frac{d^{2} q}{(2 \pi)^{2}} \frac{\mathrm{e}^{i q(x-z)}}{q^{2}}\left(-q_{1}+i q_{2}\right)$.

This result generalizes to other gauge groups as well.

We are primarily interested in the gauge invariant sector of the model, as it arises e.g. after the anomalies have been cancelled. The (nontrivial) dynamics in this sector is driven by the $\eta$ invariant. To keep the calculations in this writeup short, we shall fix the gauge and state the general result only at the end. We choose Landau gauge, $\partial \cdot A^{0}(x)=0$. In this case eq. (38) simplifies to

$$
\begin{aligned}
& f(x, y)=\exp \left\{i \int \frac{d^{2} q}{(2 \pi)^{2}} \frac{\left(\mathrm{e}^{i q x}-\mathrm{e}^{i q y}\right)}{q^{2}}\right. \\
& \left.\times\left(-q_{1} A_{2}^{0}(q)+q_{2} A_{1}^{0}(q)\right)\right\} .
\end{aligned}
$$

On the torus (or any other compact space) the momenta are discrete. So, whenever we write an integral over internal momenta, the appropriate sum respecting the boundary conditions should actually be understood. In our case it is, however, simpler to do the integrals rather than the corresponding sums. In the infinite volume limit both methods should give the same result.

We are now ready to compute the effective action. We choose the point-splitting method to regularize the integrals, which is computationally simple. Using the Pauli-Villars regularization gives the same result. It would have been even more appropriate to use the lattice regularization as proposed in sec. 3 , in particular as 
the continuum gauge fields can be stated explicitly in this case [9]. However, it is hopeless to find a closed form for the inverse of the lattice Dirac operator, which means that one has to do the sums numerically. We shall return to that as a check of the method - when we will apply our ideas to four-dimensional models. But we believe that the results will be the same. Writing now ${ }^{t} A=i t A^{0}$, we have

$$
\begin{aligned}
& \operatorname{Im} S_{-}(A)=\lim _{\epsilon_{1}, \epsilon_{2} \rightarrow 0} \frac{1}{2 \pi^{2}} \int_{0}^{1} d t \int d^{2} x \\
& \times \int d^{2} y \delta_{\epsilon_{1}, \epsilon_{2}}(x, y) \exp \left\{i \int_{y}^{x} d s_{\mu} t A_{\mu}^{0}\right\} \\
& \times \operatorname{Tr} i \gamma_{5} A^{0}(x) \int \frac{d^{2} k}{(2 \pi)^{2}} \frac{\mathrm{e}^{i k(x-y)}}{k^{2}} \\
& \times \not k\left(\begin{array}{c}
f^{*-t}(x, y) \\
\left.f(x, y)^{t}\right),
\end{array}\right.
\end{aligned}
$$

where

$$
\begin{aligned}
& \delta_{\epsilon_{1}, \epsilon_{2}}(x, y) \\
& =\frac{\epsilon_{1}}{\left(x_{1}-y_{1}\right)^{2}+\epsilon_{1}^{2}} \frac{\epsilon_{2}}{\left(x_{2}-y_{2}\right)^{2}+\epsilon_{2}^{2}} .
\end{aligned}
$$

The integral over $k$ can be done immediately and gives

$$
\frac{i}{2 \pi} \frac{\not x-\not y}{(x-y)^{2}}
$$

For the integral over $y$ only the region $y \approx x$ is relevant. We may therefore expand the line integral and $f(x, y)^{t}$ about $y=x$. This gives

$$
\begin{aligned}
& \exp \left\{i \int_{y}^{x} d s_{\mu} t A_{\mu}^{0}\right\}=1+i\left(x_{1}-y_{1}\right) t A_{1}^{0}(x) \\
& +i\left(x_{2}-y_{2}\right) t A_{2}^{0}(x)+\cdots
\end{aligned}
$$

and

$$
\begin{aligned}
& f(x, y)^{t}=1+\left(x_{1}-y_{1}\right) t A_{2}^{0}(x) \\
& -\left(x_{2}-y_{2}\right) t A_{1}^{0}(x)+\cdots
\end{aligned}
$$

If we insert this now into eq. (41) we obtain

$$
\begin{aligned}
& \operatorname{Im} S_{-}(A)=-\frac{1}{4 \pi} \int_{0}^{1} d t t \int d^{2} x \operatorname{Tr} i \gamma_{5} \\
& \times\left(\begin{array}{rr}
A^{0}(x)^{2} & \\
& A^{0}(x)^{2}
\end{array}\right) \\
& =0
\end{aligned}
$$

$\left(A^{0}(x)^{2}=A_{1}^{0}(x)^{2}+A_{2}^{0}(x)^{2}\right)$. It is straightforward to repeat the calculations in an arbitrary gauge. In momentum space we find

$$
\begin{aligned}
& \operatorname{Im} S_{-}(A)=\frac{1}{2 \pi} \int \frac{d^{2} q}{(2 \pi)^{2}} \frac{1}{q^{2}} \\
& \times\left(q_{1} A_{2}^{0}(q)-q_{2} A_{1}^{0}(q)\right) q \cdot A^{0}(q),
\end{aligned}
$$

which is the anomalous term. Altogether this means that the gauge invariant sector of the chiral Schwinger model is identical with the corresponding vector theory, i.e. the massless Schwinger model. In particular it says that the $\eta$-invariant is zero.

Along the same lines we can compute the real part of the effective action,

$\operatorname{Re}\left(S_{-}(A)-S_{-}(0)\right)=\frac{1}{2}(S(A)-S(0))$.

This amounts to replacing $\gamma_{5}$ by $i \mathbf{1}$ in eq. (46), which gives

$$
\begin{aligned}
& S(A)-S(0)=-\frac{1}{2 \pi} \int_{0}^{1} d t t \int d^{2} x \\
& \times \operatorname{Tr}\left(\begin{array}{c}
A^{0}(x)^{2} \\
A^{0}(x)^{2}
\end{array}\right) \\
& =\frac{1}{2 \pi} \int d^{2} x A^{0}(x)^{2} .
\end{aligned}
$$

This is the well known result, which says that the gauge field acquires a mass 14

$$
m^{2}=\frac{e^{2}}{\pi}
$$


Note that in our notation the pure gauge field action reads $\frac{1}{4 e^{2}} \int d^{2} x F_{\mu \nu}^{2}$. It assures us that our calculations are correct. In the chiral Schwinger model the mass is $m^{2}=e^{2} / 2 \pi$ because of eq. (48). In an arbitrary gauge eq. (49) is found to be supplemented by a pure gauge term.

\section{CONCLUSIONS}

We have formulated a method, which should allow us to answer some of the principle questions in chiral gauge theories after years of fruitless attempts.

We have confronted our ideas with the chiral Schwinger model and found that the gauge invariant sector of this model equals the vector Schwinger model. This basically means that the chiral Schwinger model has no non-trivial dynamics.

In four dimensions the main task is now to evaluate eq. (16) (or any other equivalent expression for $\left.\operatorname{Im} S_{-}(A)\right)$ and demonstrate that the result converges as $a \rightarrow 0$. In a model which is anomaly-free we expect the result to be gauge invariant. If the result for $\operatorname{Im} S_{-}(A)$ turns out to be small, we can then think of numerical simulations. If, however, $\operatorname{Im} S_{-}(A)$ is large and strongly fluctuating, we do not see any chance for lattice simulations at the moment. We like to emphasize that the problem of complex action is inherent in any other approach. Whether we can make a statement as to whether $\operatorname{Im} S_{-}(A)$ is non-zero or not remains to be seen.

Finally, we like to say that it might still be possible to find a "geometrical" expression for $\operatorname{Im} S_{-}(A)$ or the $\eta$-invariant, and one should search for it. This would improve the situation considerably.

\section{Acknowledgements}

One of us (G. S.) would like to thank the organizers of this Workshop for a most pleasant stay in Rome.

\section{References}

[1] For a recent review see, e.g.,

M. F. L. Golterman, Nucl. Phys. B (Proc. Suppl.) 20 (1991) 526;

see also the talks at this Workshop

[2] I. Montvay, Phys. Lett. 199B (1987) 89;

L. Lin, I. Montvay, H. Wittig and G. Münster, Nucl. Phys. B (Proc. Suppl.) 20 (1991) 601; see also the talk by I. Montvay at this Workshop

[3] L. Alvarez-Gaumé, S. Della Pietra and V. Della Pietra, Phys. Lett. 166B (1986) 177;

L. Alvarez-Gaumé and S. Della Pietra, in Recent Developments in Quantum Field Theory, eds. J. Ambjørn, B. J. Durhuus and J. L. Petersen, p. 95 (North-Holland, 1985);

S. Della Pietra, V. Della Pietra and L. AlvarezGaumé, Commun. Math. Phys. 109 (1987) 691

[4] P. Di Vecchia, K. Fabricius, G. C. Rossi and G. Veneziano, Nucl. Phys. B192 (1981) 392;

K. Ishikawa, G. Schierholz, H. Schneider and M. Teper, Phys. Lett. 128B (1983) 309

[5] M. Göckeler, A. S. Kronfeld, M. L. Laursen, G. Schierholz and U.-J. Wiese, Phys. Lett. 233B (1989) 192

[6] M. Lüscher, Commun. Math. Phys. 85 (1982) 29

[7] A. Phillips and D. Stone, Commun. Math. Phys. 103 (1986) 599

[8] M. Göckeler, M. L. Laursen, G. Schierholz and U.-J. Wiese, Commun. Math. Phys. 107 (1986) 467

[9] M. Göckeler, A. S. Kronfeld, G. Schierholz and U.-J. Wiese, HLRZ preprint 92-34 (1992)

[10] See, e.g., K. Bitar, A. D. Kennedy, R. Horsley, S. Meyer and P. Rossi, Nucl. Phys. B313 (1989) 348; M. Göckeler, R. Horsley, P. Rakow, G. Schierholz and R. Sommer, Nucl. Phys. B371 (1992) 713

[11] L. Alvarez-Gaumé, private communication 
[12] M. Göckeler, A. S. Kronfeld, M. L. Laursen, G. Schierholz and U.-J. Wiese, Nucl. Phys. B292 (1987) 349

[13] see, e.g.,

R. Jackiw and R. Rajaraman, Phys. Rev. Lett. 54 (1985) 1219;

H. O. Girotti, K. D. Rothe and H. J. Rothe, Phys. Rev. D33 (1986) 514;

N. K. Falck and G. Kramer, Ann. Phys. 176 (1987) 330;

D. Boyanovsky, Nucl. Phys. B294 (1987) 223;

C. Aragão de Carvalho, K. D. Rothe, C. A. Linhares and H. J. Rothe, Phys. Lett. 194B (1987) 539;

T. Berger, Cambridge preprint DAMTP-92-02 (1992)

[14] J. Schwinger, Phys. Rev. 128 (1962) 2425 